\title{
High resolution melting analysis: rapid and precise characterisation of recombinant influenza A genomes
}

\author{
Donata Kalthoff, Martin Beer and Bernd Hoffmann
}

\begin{abstract}
Background: High resolution melting analysis (HRM) is a rapid and cost-effective technique for the characterisation of PCR amplicons. Because the reverse genetics of segmented influenza A viruses allows the generation of numerous influenza A virus reassortants within a short time, methods for the rapid selection of the correct recombinants are very useful.

Methods: PCR primer pairs covering the single nucleotide polymorphism (SNP) positions of two different influenza A H5N1 strains were designed. Reassortants of the two different H5N1 isolates were used as a model to prove the suitability of HRM for the selection of the correct recombinants. Furthermore, two different cycler instruments were compared.
\end{abstract}

Results: Both cycler instruments generated comparable average melting peaks, which allowed the easy identification and selection of the correct cloned segments or reassorted viruses.

Conclusions: HRM is a highly suitable method for the rapid and precise characterisation of cloned influenza A genomes.

Keywords: Influenza a virus, Highly pathogenic avian influenza virus, High resolution melting analysis

\section{Background}

Influenza A viruses (IAV) are pathogens of major importance in both public health and veterinary medicine and have a high socio-economic impact. Research has generated a substantial increase in our knowledge of influenza viruses in recent years. A technique that has revolutionised influenza research is plasmid-based reverse genetics, which enables the generation of custom-designed recombinant viruses $[1,2]$. Routine cloning procedures are the standard for manipulating the viral genome and thus facilitate basic and applied research. The comparative segment exchange between different influenza virus strains with plasmidbased sequences and the subsequent generation of designed influenza virus reassortants is a major research technique because it facilitates studies on many topics, such as the molecular basis of IAV pathogenesis.

To date, the sequencing of cloned influenza segment plasmids and generated reassortant viruses has been necessary to confirm the successful implementation of

\footnotetext{
* Correspondence: donata.kalthoff@fli.bund.de

Institute of Diagnostic Virology, Friedrich-Loeffler-Institut, Südufer 10, 17493 Greifswald-Insel Riems, Germany
}

sequence mutations. However, novel versatile technologies for the rapid and precise detection of mutations or single nucleotide polymorphisms (SNP) are currently available. In this study, we describe the application of "High-Resolution Melt (HRM) Analysis" for the verification of newly generated IAV reassortants (with mutations in a single segment) as an exemplary practical approach.

The principle of HRM analysis is based on the melting (dissociation) behaviour of DNA as it changes its transition from double- to single-strand status in the presence of a saturating fluorescent DNA-binding dye [3]. Melting analysis detects differences in the PCR amplicons that depend on the length, base composition, and strand base pairing of the amplicon. Genotyping, microbial detection, and species identification are the main fields in current HRM analysis [4-8]. Diagnostic strategies using HRM analysis have also been developed for influenza A subtyping and the detection of resistance to neuraminidase inhibitors from human samples [9-13].

Our study focused on the identification of influenza A viruses that were generated in vitro using a reverse genetics technology [14] through HRM analysis. 


\section{Results and discussion}

Figure 1 shows a comparison of the average melting peaks of the individual RNA segments of both influenza virus strains. The most distinct melting peaks were obtained for those segments with three SNPs (two cytosines and one guanine), whereas the corresponding sequence consisted of adenine and thymines, such as that obtained for segments 3 and 6 (Figure 1 and Table 1). However, as few as two SNPs within a sequence resulted in an explicit differentiation of $0.88^{\circ} \mathrm{C}$ and $0.9^{\circ} \mathrm{C}$, e.g., as obtained for segment 7 (Figure 1 and Table 1). The smallest melting peak differences were obtained for segments 2 and 8 (values $<0.49 / 0.6$, Figure 1), which exhibited four SNPs consisting of three cytosines/guanines and one adenine/thymine in one sequence compared with three adenines/thymines and one cytosine/ guanine in the other sequence. Therefore, the SNP length is less pivotal than the SNP composition for the

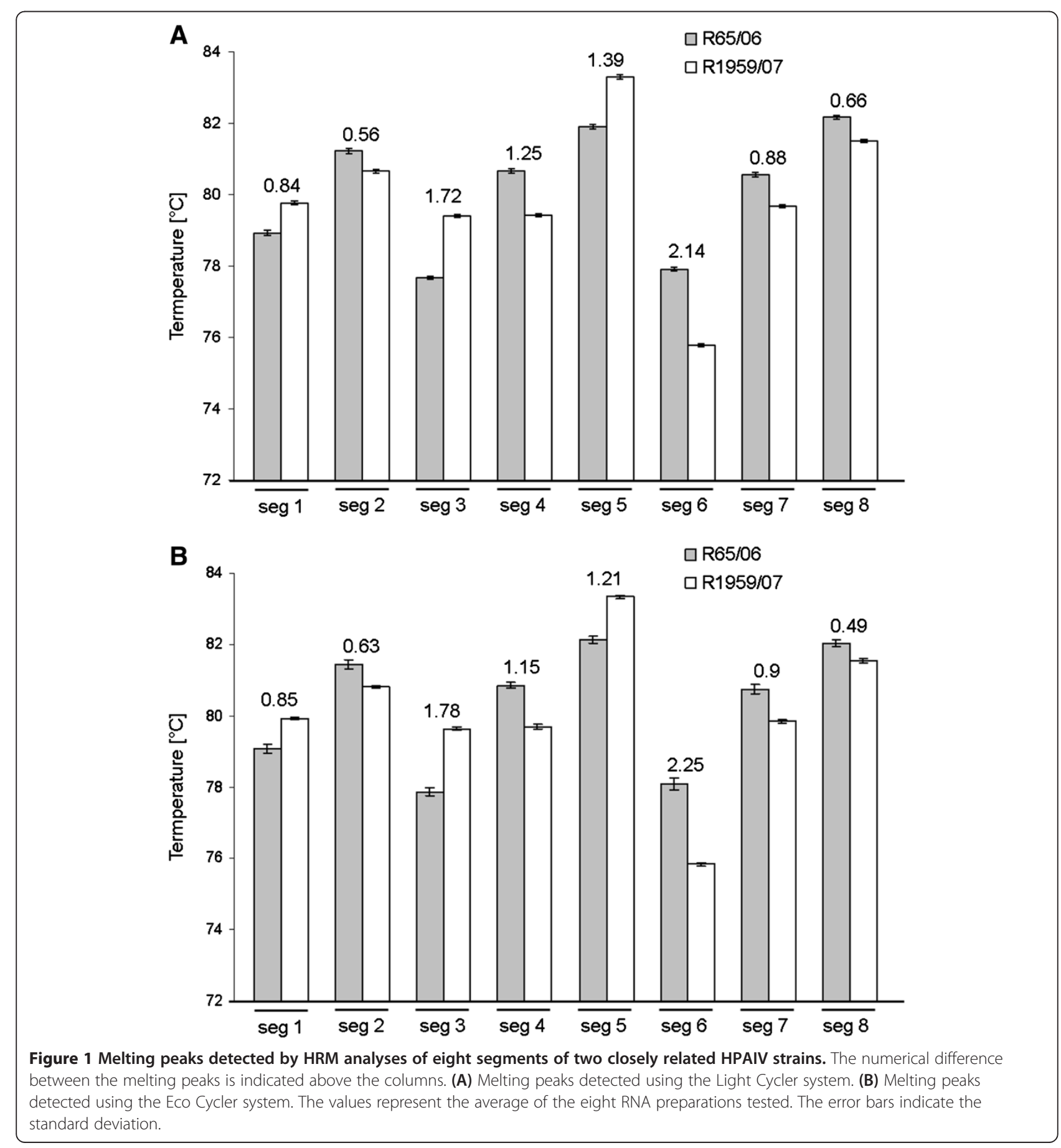


Table 1 Virus segment-specific PCR primers and corresponding SNPs

\begin{tabular}{|c|c|c|c|c|c|}
\hline & Designation & Sequence $\mathbf{5}^{\prime} \rightarrow \mathbf{3}^{\prime}$ & Amplicon length (bp) & No. of SNP & $\begin{array}{c}\text { SNPs in R65 (top row) } \\
\text { versus R1959 (bottom row) = * }\end{array}$ \\
\hline \multirow{2}{*}{ Segment 1 (PB2) } & R65-PB2-1648FW & GGTCCTGAGTCAGTGCTTG & 94 & 4 & GAAT \\
\hline & R65-PB2-1742Rv & CGGTTCAAACTCCATCTTATTGT & & & ACGG \\
\hline \multirow{2}{*}{ Segment 2 (PB1) } & R65-PB1-1524FW & ATTGTAGCCAATTCAGTATGGA & 128 & 4 & CAGG \\
\hline & R65-PB1-1652Rv & TGAAGAGCCATCTGAGCTG & & & TGTA \\
\hline \multirow{2}{*}{ Segment 3 (PA) } & R65-PA-1547Fw & GATCCCACTTGAGGAATGATAC & 78 & 3 & TTA \\
\hline & R65-PA-1625Rv & CAGTACTITTCCCACTTGTGTG & & & CCG \\
\hline \multirow{2}{*}{ Segment $4(\mathrm{HA})$} & R65-HA 204Fw & ATCTAGAYGGAGTGAAGCCTC & 85 & 3 & GCC \\
\hline & R65-HA 289Rv & TAAGACCATTCCGGCACATTG & & & ATT \\
\hline \multirow{2}{*}{ Segment 5 (NP) } & R65-NP-355Fw & TGGGTGAGAGAGCTGATTCTGTACG & 113 & 3 & ATT \\
\hline & R65-NP-468Rv & TGGAATGCCATATCATCAGGTG & & & GCC \\
\hline \multirow{2}{*}{ Segment 6 (NA) } & R65-NA-1156Fw & GGTCAGGATATAGCGGGAG & 84 & 3 & CGC \\
\hline & R65-NA-1240Rv & ATTAACTCAACCCAGAAACAAGG & & & TAT \\
\hline \multirow{2}{*}{ Segment $7(M)$} & R65-M-119Fw & TTTGCAGGAAAGAACACCGATC & 93 & 2 & CC \\
\hline & R65-M-212Rv & CAAATCCCAACATCCCTITAGTC & & & $\pi$ \\
\hline \multirow{2}{*}{ Segment 8 (NS) } & R65-NS1-224Fw & GCGAATTCTGGAGGAGGAG & 112 & 4 & CACC \\
\hline & R65-NS1-336Rv & AAGGGAACCTGTCACTTTCTG & & & TGAT \\
\hline
\end{tabular}

generation of large differences between the melting peaks of two segment sequences.

In total, 18 different viruses were engineered by reverse genetics (two ancestor viruses, eight viruses consisting of seven segments from strain R65 and one segment from strain R1959, and eight viruses consisting of seven segments from R1959 and one segment from R65). The RNA preparations of these 18 viruses were tested on both cycler systems, and all eight segments of each virus were evaluated. All of the virus strains were correctly identified using both cycler systems (Additional file 1: Table S1).

The average melting peaks detected by the Eco cycler system did not differ by more than $0.3^{\circ} \mathrm{C}$ from the average melting peaks detected with the Light cycler system. However, the melting peaks detected with the Light cycler system exhibited lower standard deviations compared with those obtained with the Eco cycler system. Nevertheless, the average differences between the melting peaks of the individual influenza segments obtained with both cycler systems exhibited very similar values (Figure 1). Although the classical approach used for the verification of recombinant influenza reassortants is based on sequencing the relevant parts of the viral genome, our approach identified the reassortant gene segment composition directly through the HRM technique. A prerequisite for this method is a set of primer pairs that cover all of the SNP positions that distinguish the viral segments. Therefore, sufficient sequence information must be available before suitable primers can be designed.

In our opinion, the use of universal primers, i.e., primers that are applicable to all influenza genomes, is unfavourable because the efforts and costs associated with the design of specific primers for each tested sequence are low. In addition primer design of universal primers that generated an amplicon suitable for HRM analysis for all divergent influenza viruses would be hard to achieve.

The virus strains tested in this study were selected because they are closely related and thus difficult to differentiate. However, the applied system discriminated the sequences correctly without any problems, and the distinction of more distantly related sequences (comprising more SNP positions) should therefore be even easier using the proposed HRM analysis. Further potential applications of this technology include the screening of genetically engineered influenza viruses to determine whether reassortment or mutation has occurred and the identification of reassortant viruses from field samples.

\section{Conclusion}

In conclusion, HRM is a valuable tool for the rapid and easy identification of reassortant influenza viruses in various settings because the total costs for genotyping by HRM are low, i.e., only a simple PCR system and a generic dye are needed.

\section{Methods}

Viruses, primers, and isolation of viral RNA

Two highly pathogenic avian influenza viruses (HPAIV) of subtype H5N1: A/swan/Germany/R65/2006 [Gisaid: EPI103081, EPI103089, EPI103087, EPI103075, EPI103085, EPI103077, EPI103079, and EPI103083] and A/Beijing duck/Germany/R1959/2007 [EPI171617, EPI171618, EPI1- 
71619, EPI171620, EPI171621, EPI171622, EPI171623, and EPI171624] were genetically engineered (13, Eck unpublished). The reassortant viruses, which were composed of seven segments of one H5N1 strain and one segment of the sister strain, were engineered; these reassortants are called " $7+1$ reassortants". Figure 2 summarises the unique segment composition of each of the 18 strains generated in this study. Stocks of the original wild-type viruses (R65/06 and R1959/07) were prepared using embryonated chicken eggs. The recombinant virus was rescued as described previously [15] and propagated using Madin-Darby canine kidney cells (MDCK, collection of cell lines in veterinary medicine, FLI Insel Riems, RIE1061). The viral RNA was extracted from the cell culture or allantoic fluid using the QIAamp viral RNA kit (Qiagen) according to the manufacturer's instructions.

\section{Primer design}

The sequence alignment of the original virus strains was used to identify the SNP positions, and specific primers were designed to cover these SNP sequences in each of the eight segments. Therefore, eight primer pairs were designed to obtain an amplicon length ranging from $78 \mathrm{bp}$ to $128 \mathrm{bp}$. The SNPs consisted of at least two and at the most four nucleotides (Table 1).

\section{Quantitative RT-PCR}

A one-step reverse transcription quantitative polymerase chain reaction (RT-qPCR) protocol was performed using the real-time ready RNA-virus-master kit (Roche Applied Science, Mannheim, Germany). The RT-qPCR assay was optimised using a total volume of $10 \mu \mathrm{l}$. Briefly, for each single PCR reaction, $5.05 \mu \mathrm{l}$ of RNase-free water, $2.0 \mu \mathrm{l}$ of $5 \times$ reaction buffer, $0.2 \mu \mathrm{l}$ of $50 \times$ enzyme-blend, $0.5 \mu \mathrm{l}$ of the primer mix (20 pmol/ $\mu \mathrm{l}$ of both primers), and $0.25 \mu \mathrm{l}$ of LightCycler ResoLight dye (Roche) were pooled to generate a master mix. Then, $2 \mu \mathrm{l}$ of the RNA template was added to each reaction. The $\mathrm{RT}-\mathrm{qPCR}$ reactions were performed on both an LightCycler 480 II instrument (Roche) and an Eco Real-Time PCR System (Illumina, San Diego, USA) using a single temperature profile: $8 \mathrm{~min}$ at $58^{\circ} \mathrm{C}$ (reverse transcription), $30 \mathrm{sec}$ at $95^{\circ} \mathrm{C}$ (inactivation reverse transcriptase/activation Taq polymerase), and 45 cycles of $1 \mathrm{sec}$ at $95^{\circ} \mathrm{C}$ (denaturation), $20 \mathrm{sec}$ at $55^{\circ} \mathrm{C}$ (annealing), and $1 \mathrm{sec}$ at $72^{\circ} \mathrm{C}$ (elongation). The quantification was analysed within each annealing step.

\section{High-resolution melting analysis}

The HRM curves were obtained by incubating the PCR products at $95^{\circ} \mathrm{C}$ for $1 \mathrm{~min}$ and then subjecting them to a renaturation step of $70^{\circ} \mathrm{C}$ for $2 \mathrm{~min}$. In the following melting step, in which the temperature was increased from $70^{\circ} \mathrm{C}$ to $90^{\circ} \mathrm{C}, 23$ fluorescence signal acquisitions per degree centigrade were detected with the Light cycler system (ramp rate of $0.02^{\circ} \mathrm{C} / \mathrm{s}$ ), whereas the Eco Cycler system detected 10 signals per degree centigrade (ramp rate of $0.08^{\circ} \mathrm{C} / \mathrm{s}$ ). The analyses of the HRM data were performed using the Light Cycler Software (Version 1.5) and the $\mathrm{Eco}^{\mathrm{TM}}$ Software (v3.0.16.0), respectively (fluorescence signal normalisation is implemented in the software).

The individual RNA segments of the 18 virus strains (reassortants and wild type) were tested on both the Light Cycler system and the Eco Cycler system. The Cqvalues determined ranged from 14.2 to 29.1, and Figure 1

\begin{tabular}{|c|c|c|c|c|c|c|c|c|}
\hline \multirow[t]{2}{*}{ Virus } & \multicolumn{8}{|c|}{ Genotype } \\
\hline & PB2 & PB1 & PA & HA & NP & NA & M & NS \\
\hline R65 parental & 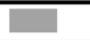 & 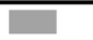 & 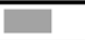 & 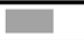 & 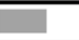 & 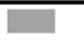 & 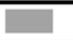 & 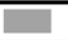 \\
\hline R1959 parental & $\square$ & $\square$ & $\square$ & $\square$ & $\square$ & $\square$ & $\square$ & $\square$ \\
\hline R65/PB2 & $\square$ & D & $\square$ & 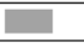 & 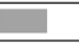 & $\square$ & $\square$ & 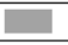 \\
\hline R65/PB1 & 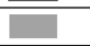 & $\square$ & 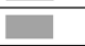 & 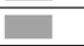 & 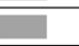 & 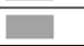 & 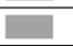 & 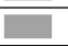 \\
\hline $\mathrm{R} 65 / \mathrm{PA}$ & 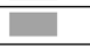 & 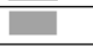 & $\square$ & 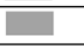 & 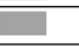 & 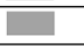 & 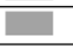 & 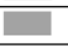 \\
\hline R65/NP & $\square$ & $\square$ & $\square$ & $\square$ & 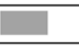 & 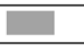 & 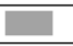 & 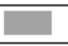 \\
\hline R65/HA & 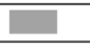 & $\square$ & 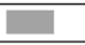 & $\square$ & $\square$ & $\square$ & 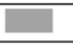 & 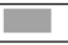 \\
\hline R65/NA & 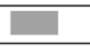 & $\square$ & $\square$ & 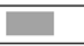 & 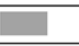 & $\square$ & $\square$ & 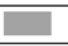 \\
\hline $\mathrm{R} 65 / \mathrm{M}$ & $\square$ & 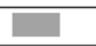 & 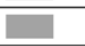 & 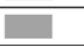 & 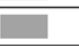 & 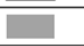 & $\square$ & 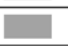 \\
\hline R65/NS & 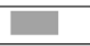 & $\square$ & 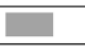 & 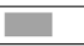 & 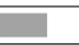 & 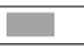 & $\bar{\square}$ & $\square$ \\
\hline R1959/PB2 & & $\square$ & $\square$ & $\square$ & $\square$ & $\square$ & $\square$ & $\square$ \\
\hline R1959/PB1 & $\square$ & 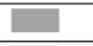 & $\square$ & $\square$ & $\square$ & $\square$ & $\square$ & $\square$ \\
\hline R1959/PA & $\square$ & $\square$ & $\square$ & $\square$ & $\square$ & $\square$ & $\square$ & $\square$ \\
\hline R1959/HA & $\square$ & $\square$ & $\square$ & 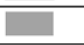 & $\square$ & 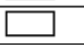 & $\square$ & $\square$ \\
\hline R1959/NP & $\square$ & $\square$ & $\square$ & $\square$ & 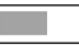 & $\square$ & $\square$ & $\square$ \\
\hline R1959/NA & $\square$ & $\square$ & $\square$ & $\square$ & $\square$ & $\square$ & $\square$ & $\square$ \\
\hline R1959/M & $\square$ & $\square$ & $\square$ & $\square$ & $\square$ & $\square$ & 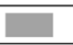 & $\square$ \\
\hline R1959/NS & $\square$ & $\square$ & $\square$ & $\square$ & $\square$ & $\square$ & $\square$ & 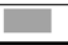 \\
\hline
\end{tabular}

Figure 2 Genomic segment composition of the analysed influenza a strains. 
shows the average value of eight replicates. The RNA (non-DNA) amplification results were verified by analysing one dataset after it was subjected to DNAse treatment (data available upon request).

Additional file 1: Table S1 presents the results of all of the analysed viral reassortants as row data, and Additional file 2: Figure S1 summarises the derivative plots for each influenza virus segment.

\section{Additional files}

Additional file 1: Table S1. Virus segment specific melting peak values. Additional file 2: Figure S1. Derivative plots representing the data for all eight influenza A virus segments. The plots were generated using the Light Cycler System.

\section{Abbreviations}

Cq: Quantification cycle; HPAIV: Highly pathogenic avian influenza virus; HRM: High-resolution melting; IAV: Influenza a virus; PCR: Polymerase chain reaction; RT: Reverse transcription; SNP: Single nucleotide polymorphisms; Tm: Melting temperature.

\section{Competing interests}

The authors declare that they have no competing interests.

\section{Authors' contributions}

$\mathrm{DK}$ and $\mathrm{BH}$ conceived, designed, and performed the experiments, and $\mathrm{DK}, \mathrm{BH}$, and $\mathrm{MB}$ analysed the data. All of the authors read and approved this manuscript.

\section{Authors' information}

Donata Kalthoff is a veterinarian at the Institute of Diagnostic Virology at Friedrich-Loeffler-Institut in Greifswald-Insel Riems. Her research interests are focused on pathogenesis and vaccine development.

\section{Acknowledgements}

We are indebted to Patrick Zitzow for the excellent technical assistance. We are also very grateful to Jürgen Stech und Melanie Eck for providing the reverse genetics plasmids. We are thankful to amplifa Labortechnik GmbH (Wasserburg, Germany) and Roche Diagnostics GmbH (Penzberg, Germany) for providing the cycler systems. This project was funded by the European Union FP7 project European Management Platform for Emerging and Re-emerging Infectious Disease Entities (EMPERIE; no. 223498).

Received: 7 May 2013 Accepted: 9 September 2013 Published: 12 September 2013

\section{References}

1. Neumann G, Watanabe T, Ito H, Watanabe S, Goto H, Gao P, Hughes M, Perez DR, Donis R, Hoffmann E, Hobom G, Kawaoka Y: Generation of influenza A viruses entirely from cloned cDNAs. Proc Natl Acad Sci USA 1999, 96:9345-50.

2. Fodor E, Devenish L, Engelhardt OG, Palese P, Brownlee GG, García-Sastre A: Rescue of influenza A virus from recombinant DNA. J Virol 1999, 73:9679-82.

3. Reed GH, Kent JO, Wittwer CT: High-resolution DNA melting analysis for simple and efficient molecular diagnostics. Pharmacogenomics 2007, 8:597-608.

4. Costa JM, Cabaret O, Moukoury S, Bretagne S: Genotyping of the protozoan pathogen toxoplaasma gondii using high-resolution melting analysis of the repeated B1 gene. J Microbiol Methods 2011, 86:357-63.

5. Fortini D, Ciammaruconi A, De Santis R, Fasanella A, Battisti A, D'Amelio R, Lista F, Cassone A, Carattoli A: Optimization of high-resolution melting analysis for low-cost and rapid screening of allelic variants of bacillus anthracis by multiple-locus variable-number tandem repeat analysis. Clin Chem 2007, 53:1377-80.

6. Giglio S, Monis PT, Saint CP: Legionella confirmation using real-time PCR and SYTO9 is an alternative to current methodology. Appl Environ Microbiol 2005, 71:8944-8.
7. Patel KP, Barkoh BA, Chen Z, Ma D, Reddy N, Medeiros LJ, Luthra R: Diagnostic testing for IDH1 and IDH2 variants in acute myeloid leukemia an algorithmic approach using high-resolution melting curve analysis. J Mol Diagn 2011, 13:678-86.

8. Wittwer CT, Reed GH, Gundry CN, Vandersteen JG, Pryor RJ: High-resolution genotyping by amplicon melting analysis using LCGreen. Clin Chem 2003, 49:853-60.

9. Lin JH, Tseng CP, Chen YJ, Lin CY, Chang SS, Wu HS, Cheng JC: Rapid differentiation of influenza $A$ virus subtypes and genetic screening for virus variants by high-resolution melting analysis. J Clin Microbiol 2008, 46:1090-7.

10. Varillas D, Bermejo-Martin JF, Almansa R, Rojo S, Nogueira B, Eiros JM, Rico L, Iglesias V, Lejarazu RO: A new method for detection of pandemic influenza virus using high resolution melting analysis of the neuraminidase gene. J Virol Methods 2010, 171:284-6.

11. Tong SY, Dakh F, Hurt AC, Deng YM, Freeman K, Fagan PK, Barr IG, Giffard PM Rapid detection of the $\mathrm{H} 275 \mathrm{Y}$ oseltamivir resistance mutation in influenza A/H1N1 2009 by single base pair RT-PCR and high-resolution melting. PLoS One 2011, 6:e21446.

12. Chen N, Pinsky BA, Lee BP, Lin M, Schrijver I: Ultrasensitive detection of drug-resistant pandemic 2009 (H1N1) influenza A virus by rare-variant-sensitive high-resolution melting-curve analysis. J Clin Microbiol 2011, 49:2602-9.

13. Lee HK, Lee CK, Loh TP, Tang JW, Tambyah PA, Koay ES: High-resolution melting approach to efficient identification and quantification of $\mathrm{H} 275 \mathrm{Y}$ mutant influenza $\mathrm{H} 1 \mathrm{~N} 1 / 2009$ virus in mixed-virus-population samples. J Clin Microbiol 2011, 49:3555-9.

14. Stech J, Stech O, Herwig A, Altmeppen H, Hundt J, Gohrbandt S, Kreibich A, Weber S, Klenk HD, Mettenleiter TC: Rapid and reliable universal cloning of influenza A virus genes by target-primed plasmid amplification. Nucleic Acids Res 2008, 36:e139.

15. Gabriel G, Dauber B, Wolff T, Planz O, Klenk HD, Stech J: The viral polymerase mediates adaptation of an avian influenza virus to a mammalian host. Proc Natl Acad Sci USA 2005, 102:18590-18595.

doi:10.1186/1743-422X-10-284

Cite this article as: Kalthoff et al:: High resolution melting analysis: rapid and precise characterisation of recombinant influenza A genomes. Virology Journal 2013 10:284.

\section{Submit your next manuscript to BioMed Central and take full advantage of:}

- Convenient online submission

- Thorough peer review

- No space constraints or color figure charges

- Immediate publication on acceptance

- Inclusion in PubMed, CAS, Scopus and Google Scholar

- Research which is freely available for redistribution 